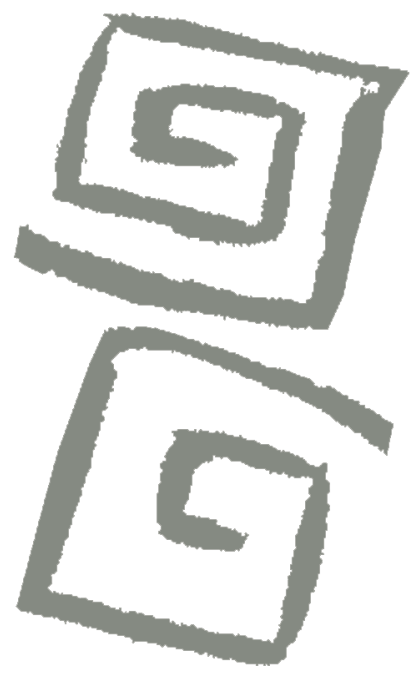

\title{
"Parir no es un asunto de etnia, es un asunto de humanidad": experiencias frente a la violencia obstétrica durante la atención al parto en mujeres indígenas
}

\author{
"Giving birth is not a matter of ethnicity, it is a matter \\ of humanity": experiences of obstetric violence during \\ childbirth among indigenous women
}

Emily Gaffney Gleason ${ }^{1}$, Diana Patricia Molina Berrío², Jennifer Marcela López Ríos $^{3}$, Cristina María Mejía Merino ${ }^{4}$

${ }^{1}$ Bachelor of Arts en Antropología. Perelman School of Medicine, University of Pennsylvania, Philadelphia, EEUU. $\triangle$ iD

${ }^{2}$ Magíster en Salud Pública. Profesora e investigadora, Universidad de Antioquia, Medellín, Colombia. $\bowtie$ (iD)

${ }^{3}$ Magíster en Salud Pública. Profesora e investigadora, Universidad de Antioquia, Medellín, Colombia. $₫$ (iD

${ }^{4}$ Magíster en Salud Pública. Coordinadora Componente de Nutrición, Ruta de Promoción y Mantenimiento de la Salud, Programa de Salud, Universidad de Antioquia, Medellín, Colombia. $₫$ iD
RESUMEN El objetivo de este estudio es comprender las experiencias frente a la violencia obstétrica que vivieron las mujeres emberas durante la atención del parto en servicios de salud de la ciudad de Medellín, Colombia. A través de un abordaje cualitativo de tipo etnográfico, con entrevistas semiestructuradas realizadas a nueve mujeres indígenas emberas entre febrero y marzo de 2020, se identificó que estas mujeres, representantes del grupo más grande de los pueblos indígenas en Medellín, experimentan formas de violencia obstétrica similares a las vividas por mujeres occidentalizadas, que pueden ser explicadas por el habitus médico autoritario, y por un sistema de salud que privilegia las lógicas del mercado. Sin embargo, esta investigación amplía la comprensión de la violencia obstétrica al identificar que las mujeres indígenas experimentan otra forma particular de este fenómeno: la falta de respeto o sensibilidad cultural, lo cual deviene del proceso de colonialidad vivido por los pueblos originarios, y que hoy en día se expresa en el contexto de la atención al parto, bajo formas como el desconocimiento y desprecio de los saberes ancestrales que las mujeres de las comunidades indígenas aún conservan y que podemos comprender como microagresiones comunes y ambiguas.

PALABRAS CLAVES Deshumanización; Parto Humanizado; Pueblos Indígenas; Personal de Salud; Colombia.

\begin{abstract}
This study aims to understand the experiences of obstetric violence experienced by Embera women during childbirth at healthcare facilities in the city of Medellin, Colombia. Employing a qualitative, ethnographic approach, semi-structured interviews were conducted with nine indigenous Embera women between February and March of 2020. The data indicate these women, members of the largest indigenous community in Medellín, experience similar forms of obstetric violence to non-indigenous women, which might be explained by the authoritarian medical habitus and a health system that prioritizes market forces. However, the data also reveal that indigenous women experience a specific form of this phenomenon: a lack of respect or cultural sensitivity, stemming from the process of colonialism to which indigenous peoples have been subjected. This dynamic continues to be expressed during delivery in healthcare childbirth settings via ignorance and disregard for indigenous ancestral knowledge, which can be understood as microaggressions that occur frequently but are difficult to identify.

KEY WORDS Dehumanization; Humanizing Delivery; Indigenous Peoples; Health Personnel; Colombia.
\end{abstract}




\section{INTRODUCCIÓN}

El desarrollo de los programas de salud, especialmente en lo relacionado con la salud reproductiva y la salud sexual, es crucial para proteger los derechos de las personas al bienestar y a la dignidad básica, en tanto la reproducción es quizás uno de los aspectos más críticos a considerar en un enfoque de la salud basado en los derechos humanos, porque afecta toda la vida y bienestar de la mujer ${ }^{(1)}$.

La atención a la salud materna es uno de los componentes del objetivo de desarroIlo sostenible "salud y bienestar", el cual ha inspirado múltiples acciones para mejorar la atención para todas las mujeres; sin embargo, la morbilidad y la mortalidad materna no han sido erradicadas y todavía existen otros problemas con el acceso a un parto sano y seguro. Uno de ellos es la violencia obstétrica, entendida como una forma de violencia que se ejerce en el sistema de salud, ya sea intencionada o involuntariamente, contra las mujeres gestantes ${ }^{(2,3)}$, convirtiéndose a su vez, en una intersección inherente entre la violencia institucional y la violencia de género contra las mujeres.

Una investigación realizada por Mejía y colaboradores indica que existe un desconocimiento de los temas de violencia obstétrica, los derechos sexuales y los derechos reproductivos, durante la formación de los profesionales de salud ${ }^{(4)}$. De acuerdo a los autores, las experiencias de las mujeres existen en un plano de importancia secundaria al bienestar físico, un plano al que tal vez las instituciones del sistema de salud no pueden acceder debido a las brechas en la comprensión, los antecedentes culturales y la confianza ${ }^{(4)}$. Sin embargo, muchas mujeres identifican elementos de violencia obstétrica en la atención de salud materna. Estos aspectos actúan como fuertes elementos disuasorios que impiden que estas mujeres y otras personas en sus redes sociales utilicen la atención especializada de los hospitales, por lo que se configuran en problemas importantes de la calidad de la atención ${ }^{(5)}$.

Las mujeres indígenas, particularmente, enfrentan barreras únicas para utilizar y acceder a la atención de salud materna, porque son un grupo especialmente vulnerable: tienen más probabilidades de tener un estatus socioeconómico bajo, corren un mayor riesgo de sufrir trauma psicológico después de su desplazamiento (debido al apego histórico a sus tierras) y enfrentan obstáculos culturales únicos en la búsqueda y recepción de atención ${ }^{(6)}$. A esto se le adiciona el maltrato cultural presente en la salud reproductiva, que también afecta la labor de las parteras tradicionales dado que, por los prejuicios existentes en nuestra sociedad mestiza contemporánea, no les dan la oportunidad de demostrar sus aportes y prácticas, recibiendo rechazo y maltrato del personal de $\operatorname{salud}^{(7)}$.

En las últimas décadas, el estudio alrededor de la violencia obstétrica y el parto humanizado se ha convertido en un discurso académico crítico, tanto en el mundo -COnocido en otros lugares más por el término de falta de respeto y abuso, disrespect and abuse (D\&A), que por violencia obstétrica-, como en América Latina( ${ }^{(8)}$. Estudios internacionales como el de Sadler et al. expresan que existe una preocupación por parte de algunos investigadores debido al exceso de las intervenciones médicas y la falta de respeto hacia las mujeres durante el parto, como consecuencia de la violencia estructural que se viene presentando históricamente en la atención de la maternidad ${ }^{(9)}$. Asimismo, en una revisión sistemática de métodos mixtos se encontró que las experiencias de parto de las mujeres en todo el mundo se ven empañadas por el maltrato y que, "a pesar del creciente reconocimiento del trato negligente, abusivo e irrespetuoso hacia las mujeres durante el parto en los establecimientos de salud, no existe un consenso a nivel mundial sobre cómo se definen y se miden estos sucesos"(10).

En Latinoamérica, uno de los estudios realizado en un hospital público del Estado de Morelos, en México, muestra que las formas de violencia obstétrica que son más comunes y las que más padecen las mujeres en las salas de parto son: a) el abuso físico, traducido en cantidad excesiva y brusquedad en los tactos vaginales realizados por los profesionales de la salud; b) abuso verbal, representado en 
comentarios denigrantes y sexistas, además de manifestaciones de burla; c) ausencia de información y del consentimiento informado en la atención del parto, que genera confusión y malas interpretaciones por parte de las mujeres y poca comprensión de los procedimientos o maniobras a las son sometidas durante el parto; d) abandono de la mujer, que se expresa en ignorar los llamados y las solicitudes de esta ${ }^{(11)}$. En Colombia, por su parte, un estudio realizado por Restrepo et al., encontró que, aunque la violencia obstétrica es un fenómeno que "marca a las mujeres durante toda su vida y puede influir negativamente en su deseo de tener más hijos", sigue siendo un tema desconocido, ignorado o incluso naturalizado, tanto por las mujeres, como por el personal de salud, lo que exige una mayor visibilidad y relevancia en las agendas públicas del gobierno y la academia $^{(12)}$. En Perú, el informe con enfoque de género sobre maltrato y violencia obstétrica contra las mujeres indígenas, durante la atención de salud reproductiva, reveló que pese a que la violencia obstétrica entró a formar parte de las reflexiones del país, en materia de salud sexual y salud reproductiva, siguen aumentando los casos de discriminación, maltrato, abuso e irrespeto en la atención a las mujeres, especialmente indígenas, en las diferentes etapas del embarazo, lo que pone de manifiesto un claro incumplimiento de los derechos humanos de las mujeres, el derecho a una vida libre de violencia y, por tanto, a la vulneración de los derechos contemplados en la Constitución ${ }^{(13)}$.

Sin embargo, pese a los múltiples estudios que versan sobre el tema, en los últimos treinta años, el acervo documental alrededor de la violencia obstétrica o D\&A en las comunidades indígenas es limitado ${ }^{(14)}$. Los estudios realizados en México indican que las mujeres indígenas son la población que menos se queja de las experiencias de maltrato o abuso durante el parto y la que tiene menos conocimiento sobre sus derechos a un parto seguro, autónomo y humanizado ${ }^{(14)}$.

Las mujeres indígenas, que han emigrado a nuevos contextos urbanos, tienen un riesgo mayor de experimentar la violencia obstétrica, debido a sus vulnerabilidades (como la pobreza, el idioma, los desafíos en navegar un contexto nuevo, etc. $)^{(15)}$. Sin embargo, este fenómeno ha sido históricamente invisibilizado por las instituciones de salud y por el discurso académico ${ }^{(16)}$. Además de los factores típicos considerados en los análisis de la violencia obstétrica (como la hegemonía biomédica, la naturalización de la violencia institucionalizada, la falta de un discurso de derechos humanos en las formaciones académicas en Medicina, etc.), las mujeres indígenas también se enfrentan al desafío único de la interculturalidad y el conflicto entre la medicina tradicional y la occidental ${ }^{(17)}$.

La comunidad indígena embera representa el grupo más grande de indígenas en Medellín ${ }^{(18)}$. Es una comunidad conformada aproximadamente por 42.000 personas en el país, divididos en tres subgrupos: Embera Chamí ("gente de la montaña"), Embera Dobidá ("gente de río") y Embera Eyabida o Katío ("gente de la selva") $)^{(19)}$. Su lengua tradicional es el embera y sus familias son extensas, es decir, con múltiples generaciones viviendo juntas ${ }^{(20)}$. Para esta comunidad, el embarazo y la maternidad son de especial importancia no solo por la existencia de prácticas y tradiciones culturales específicas en torno al parto ${ }^{(21)}$, sino también porque son maneras de continuación étnica y de reproducción social y cultural ${ }^{(22,23)}$.

Partiendo de la premisa de que los saberes y prácticas tradicionales afectan gran parte de la experiencia de atención de la salud materna de las mujeres indígenas, y que incorporar una perspectiva multicultural puede contribuir a buscar formas más respetuosas y compasivas de abordar el embarazo, el parto y posparto, el presente estudio busca comprender las experiencias frente a la violencia obstétrica que vivieron las mujeres emberas durante la atención del parto en servicios de salud de la ciudad de Medellín, Colombia, poniendo en evidencia un fenómeno frente al cual es urgente su reconocimiento para pensar en estrategias que transformen esta realidad.

Cabe señalar que los resultados de investigación que se exponen a continuación 
hacen parte de una investigación mayor cuyo título es "La salud materna y la violencia obstétrica: las experiencias de las mujeres emberas en Medellín - Colombia, 2019-2020". Para este artículo solo se presentan las categorías que recogen las experiencias frente a la violencia obstétrica.

\section{METODOLOGÍA}

Se llevó a cabo un estudio cualitativo de tipo etnográfico ${ }^{(24)}$ y se utilizaron herramientas analíticas de la teoría fundamentada ${ }^{(25)}$, la cual propone examinar sistemáticamente los datos recogidos para generar teorías descriptivas, a través de la identificación de temas y módulos comunes, y la agrupación de temas en categorías conceptuales más amplias.

\section{Recolección de información y selección de participantes}

Para la recolección de información se utilizó la técnica de entrevista semiestructurada, la cual se entiende como "una forma específica de interacción social, que tiene por objeto recolectar datos para una investigación"(26) y permite flexibilidad en la interacción con los participantes al alternar preguntas previamente establecidas y preguntas espontáneas resultantes de la conversación.

Entre febrero y marzo de 2020 se entrevistaron nueve mujeres indígenas emberas habitantes de la ciudad de Medellín. Estas mujeres fueron contactadas a través de la Organización Indígena de Antioquia, entidad aliada en las diferentes fases de este proyecto de investigación y encargada de agrupar, proteger y defender las comunidades indígenas del departamento ${ }^{(27)}$. Se eligió este número de participantes no solo por la viabilidad del marco temporal y de los recursos disponibles para el proyecto ${ }^{(28)}$, sino también porque con las entrevistas realizadas se logró la saturación teórica de las categorías ${ }^{(29,30)}$. Los criterios de inclusión establecidos para la participación de las mujeres fueron: dar su consentimiento informado para participar en el proyecto, tener más de 14 años, autoreconocerse como indígena embera, hablar español y haber tenido al menos una experiencia de atención durante el parto en un servicio de salud institucionalizado de la ciudad de Medellín.

Las participantes fueron seleccionadas a través de la técnica de bola de nieve. La recolección de información fue realizada por la investigadora principal del proyecto y una psicóloga embera, quien estuvo presente para brindar atención psicológica a las mujeres que presentaran alguna respuesta crítica emocional durante la entrevista. Cada una de las entrevistas tuvo una duración promedio de 30 minutos. Éstas fueron grabadas en audio y posteriormente transcritas literalmente. Para ello se utilizó un guion de entrevista de 15 preguntas.

Las entrevistas fueron semiestructuradas, lo que significa que se hicieron las mismas preguntas a todas las participantes, pero se dejó espacio para los matices y la discusión posterior según las experiencias de cada mujer. Aunque estas técnicas cualitativas permiten cierto grado de subjetividad en las entrevistas, nos basamos en gran medida en el Ilamamiento a reconceptualizar la idea de sesgo en la investigación cualitativa y no equiparar el fenómeno con la subjetividad (31). Acogimos la subjetividad introducida por cada mujer, y el uso de preguntas claves nos permitió elaborar plenamente las diferencias y similitudes entre las experiencias de parto de las participantes. Los temas claves que se trataron fueron los siguientes: 1) información demográfica; 2) accesibilidad a la atención prenatal y de maternidad; 3) experiencia(s) de parto, incluyendo lugar, año, nivel de comodidad, experiencias positivas o negativas, y posición; 4) uso de curanderos o medicina tradicional; 5) trato recibido por parte de los profesionales de salud; 6) problemas o complicaciones durante el parto; 7) experiencias de violencia obstétrica; y 8) recomendaciones para mejorar la experiencia del parto en el hospital. 


\section{Análisis de información}

Se utilizaron las herramientas analíticas de la codificación y la categorización propuestas por la teoría fundamentada ${ }^{(32)}$. Para este proceso se utilizó el software de análisis cualitativo NVivo versión 12, en dos fases: en la primera, se realizó la codificación abierta, en la cual se explica cómo los fenómenos se relacionan entre sí y cuyo proceso derivó en la conformación de unas categorías descriptivas; en la segunda, conocida como codificación axial, se reagruparon los datos obtenidos en la codificación abierta en categorías analíticas, lo que posibilitó una presentación sintética de los hallazgos ${ }^{(32)}$. Se logró la saturación de información ${ }^{(33)}$ en cinco categorías, que son las que se presentan en el presente artículo. Aquí cabe precisar que, si bien la participación de nueve informantes es un grupo pequeño, otros autores expertos en estudios cualitativos, como Saunders y Bernard, afirman que es posible lograr la saturación de categorías con muestras pequeñas ${ }^{(29,30)}$, tal y como sucedió en nuestro estudio. Los hallazgos descritos en este manuscrito fueron validados por las participantes y el consejero del Programa de Salud Propia e Intercultural de la Organización Indígena de Antioquia.

\section{Limitaciones}

Este estudio tiene como limitación que las mujeres indígenas participantes del estudio hablaban un español fluido y la mayoría de ellas trabajaba o estudiaba en Medellín, lo que supone un proceso importante de aculturación. En este sentido, consideramos que se habría podido obtener otros resultados u otras categorías de análisis, si se hubiese contado con la participación de mujeres con poco contacto con la cultura occidental.

\section{Aspectos éticos}

Este artículo se deriva del proyecto de investigación "La salud materna y la violencia obstétrica: las experiencias de las mujeres emberas en Medellín-Colombia, 2019-2020" el cual está avalado por el Comité de Ética de Investigación, de la Facultad Nacional de Salud Pública de la Universidad de Antioquia, en el acta 21030002-00318-2019, sesión 220 del 11 de octubre del 2019. Para esta investigación se siguieron los requerimientos de la declaración de Helsinki ${ }^{(34)}$ y las Normas Científicas, Técnicas y Administrativas para la Investigación en Salud, según la Resolución 008430, del 4 de octubre de 1993, del Ministerio de Salud de Colombia ${ }^{(35)}$, clasificada como investigación con riesgo mínimo. Todas las mujeres participantes del estudio dieron su consentimiento informado verbal. Solo las investigadoras tuvieron acceso a la información obtenida.

\section{RESULTADOS}

Para las entrevistas, se seleccionaron nueve mujeres indígenas de diversas etnias y de diversos municipios de la Nación Embera radicadas en el municipio de Medellín, las cuales habían experimentado al menos un parto institucional. En la Tabla 1 se describen algunas características de las mujeres participantes.

El análisis de los datos ha revelado que las mujeres de este estudio experimentaron una violencia obstétrica que varió tanto en

Tabla 1. Características demográficas de las mujeres indígenas participantes, Medellín, Colombia, 2020.

\begin{tabular}{|l|c|l|l|l|}
\hline N & $\begin{array}{l}\text { Edad (en el } \\
\text { momento de } \\
\text { la entrevista) }\end{array}$ & Etnia & $\begin{array}{l}\text { Municipio } \\
\text { de origen }\end{array}$ & $\begin{array}{l}\text { Años desde } \\
\text { el parto más } \\
\text { reciente }\end{array}$ \\
\hline Mujer 1 & 43 & Emberá Chamí & Jardín & 13 \\
\hline Mujer 2 & 45 & Emberá Chamí & Jardín & 13 \\
\hline Mujer 3 & 37 & Emberá Chamí & Jardín & 15 \\
\hline Mujer 4 & 40 & Emberá Chamí & Jardín & 15 \\
\hline Mujer 5 & 33 & Emberá Chamí & Turbo & 15 \\
\hline Mujer 6 & 32 & Emberá Chamí & Jardín & 2 \\
\hline Mujer 7 & 37 & Emberá Eyabida & Frontino & 4 \\
\hline Mujer 8 & 48 & Emberá Eyabida & Mutatá & 25 \\
\hline Mujer 9 & 59 & Emberá Chamí & Jardín & 26 \\
\hline
\end{tabular}

Fuente: Elaboración propia. 
su forma, como en su gravedad. Describimos cinco categorías de violencia obstétrica: sentirse ignorada o descuidada; falta de respeto o sensibilidad cultural; falta de consentimiento informado; atención sin dignidad (incluyendo lo que algunos académicos describen como abuso verbal); y abuso físico. Cuatro de estas categorías se basaron en los marcos comúnmente utilizados en los tipos de falta de respeto y abuso que pueden ocurrir durante el parto, según lo establecido por d'Oliveira et al. ${ }^{(36)}$, Bowser y Hill(37), y Castro y Erviti ${ }^{(38)}$, y ligeramente modificados para captar los tipos de violencia obstétrica más frecuentemente experimentados por las mujeres de este estudio. La otra categoría, falta de respeto o sensibilidad cultural, fue creada por las autoras del estudio, para referirnos al rechazo o la ignorancia de los conocimientos, prácticas y saberes tradicionales que experimentan las mujeres indígenas en relación con el embarazo y el parto. Esta forma de violencia en particular llevó a experiencias de desconfianza, alienación y sentimientos de depresión entre las mujeres del estudio.

Además de capturar los diversos tipos de violencia obstétrica experimentada por las mujeres, intentamos retratar el amplio espectro de la gravedad de las violencias que pueden ocurrir; entendiendo que las manifestaciones de esta pueden interpretarse como superpuestas y no mutuamente excluyentes. Algunas de las siguientes experiencias de violencia obstétrica son flagrantes y chocantes, mientras que otras se encuentran profundamente naturalizadas. Consideramos que destacar todos los casos de esta forma de violencia, incluso los "menos graves", es fundamental para comprender el fenómeno y afrontarlo activamente.

\section{Sentirse ignorada o descuidada}

Ocho de las nueve mujeres entrevistadas describieron sentirse ignoradas frente a sus dudas y demandas de atención que el ritmo del trabajo de parto las obligaba realizar. Los relatos también evidenciaron negligencia por parte de los profesionales de la salud, al pedirles que intentaran contener el proceso de parto, a pesar de la solicitud expresa de las madres de sentir el bebé en camino, situación que las mujeres experimentaron con pánico y angustia, al sentir que sus preocupaciones y el saber que ellas tenían del proceso que estaban viviendo, fue desestimado e ignorado.

Llamé a un enfermero y le dije "chico me estoy sintiendo así y tengo mucho dolor, tengo muchas contracciones muy seguidas... el chico me dijo "¿y tú cómo sabes que son contracciones?", yo le decía "yo soy la que la está cargando y yo sé que tengo contracciones... me estás poniendo en riesgo mi vida y la de mi hijo, llámame a la cirujana, llámame a la médica, a la ginecobstetra que me atendió". Nunca lo hizo. Yo le digo a mi hermana "yo no me siento bien, yo siento que yo voy a entrar en proceso de parto, búsquese a mi médica y llámela", ella corrió, buscó, la encontró, no sé cómo lo hizo, pero lo hizo. Me dice: ¡no, Dios mío! ¡Todo se complicó! (Mujer 4, 40 años)

Otras mujeres describieron casos de negligencia flagrante, como aquellos en los cuales, a mujeres indígenas, prontas a realizar su trabajo de parto, se les negó la posibilidad de ingresar al hospital bajo argumentos como no estar afiliadas a una Entidad Promotora de Salud (EPS) o no tener camillas disponibles. Estas situaciones llevaron a las mujeres a realizar su parto en la entrada de un quirófano o peor aún, en la puerta del hospital.

\section{Falta de respeto o sensibilidad cultural}

Todas las mujeres indígenas entrevistadas en este estudio coincidieron en que las instituciones prestadoras de servicios de salud y los profesionales de medicina occidental no reconocían ni respetaban los saberes, prácticas y conocimientos de las comunidades indígenas y su medicina tradicional. Muchas de ellas experimentaron el rechazo de sus 
saberes o la falta de integrar sus prácticas y rituales en el entorno hospitalario. Otras dijeron que los profesionales de la salud a menudo no les preguntaban -o no podían hacerlo si la mujer no hablaba español- sobre sus preferencias culturales en cuanto al parto. Esta situación se evidenció especialmente en la imposibilidad que tuvieron las mujeres al no poder acceder a que les entregaran su placenta. Las mujeres de estas comunidades tienen un ritual significativo que consiste en enterrar dicho órgano para crear vínculo entre el alma de su hijo o hija y su territorio. Sin embargo, a las emberas que tuvieron sus hijos en espacios institucionalizados se les negó el derecho de realizar este ritual, debido a la negativa de los profesionales de salud, quienes desconocen o ignoran sus solicitudes, impidiéndoles acceder a su propia placenta. Otros rituales que fueron rechazados incluyeron: la presencia de las parteras -curanderas tradicionales que suelen acompañarlas durante todo el embarazo-durante el parto; la realización de baños tradicionales con frutos sagrados y agua caliente; permitir que la mujer pueda cortar las uñas de su propio hijo por primera vez; y beber tés calientes y caldos tradicionales durante el trabajo de parto:

Yo me enojé con la pediatra porque el niño nació con las uñas largas y se las cortó, porque para nosotros hay un ritual, porque son las primeras uñitas, hay que conseguirle madrina, era un ritual y entonces... pensé que se lo habían Ilevado a cambiarlo, a ponerle la ropita y cuando yo alcanzo a ver... dije: “ay, no, porqué me le cortó las uñas, no ve que necesita un ritual, si no usted me las tiene que pagar", entonces ella me dijo: "si con esas uñas iba a romper la placenta", eso fue la respuesta que me dio. (Mujer 6, 32 años)

El ambiente del hospital también fue visto como violento por muchas mujeres, porque difiere significativamente con sus preferencias culturales. El aire frío, las paredes blancas y los gritos que se escuchaban de otras mujeres en trabajo de parto en la misma habitación, separadas solo por cortinas, contribuyen a un ambiente que se siente hostil y extraño. Además, los hospitales nunca estaban equipados para atender a las mujeres indígenas que no hablaban español. Varias participantes informaron que no había servicios de intérprete o ni siquiera se ofrecían, por lo que era imposible que estas mujeres recibieran una atención equitativa y diferencial. Adicionalmente, las participantes indicaron que a menudo se utilizaba la terminología médica sin explicación, lo que impedía a las mujeres participar activamente en su propia atención.

\section{Falta de consentimiento informado}

Varias mujeres describieron que se sentían insuficientemente informadas sobre el proceso de parto o los procedimientos a los que se sometían en el hospital. Ellas señalaron que, como indígenas, ingresaron al sistema hospitalario con una falta de conocimiento sobre la medicina occidental, las prácticas hospitalarias y sus derechos en el ámbito de la atención en salud. Esto las dejó sintiéndose asustadas, perdidas y confundidas mientras recibían atención, y los profesionales de la salud rara vez se dieron cuenta o se tomaron el tiempo de explicarles los procedimientos. Incluso cuando algo durante el nacimiento salió mal o cuando, trágicamente, el niño murió durante el nacimiento, el personal del hospital nunca explicó a la mujer lo que había ocurrido:

$$
\begin{aligned}
& \text { Nadie me dijo nada [después del } \\
& \text { aborto]... me llevan para la habitación, } \\
& \text { cuando yo llego a la habitación, hasta } \\
& \text { ese punto nadie me dice nada ¿Qué } \\
& \text { pasó? ¡Mi bebé! ¿Sobrevivió? Nadie me } \\
& \text { decía nada ¿Cómo me enteré yo? Por mi } \\
& \text { familia, esa fue la manera de yo ente- } \\
& \text { rarme que mi hija no sobrevivió al parto. } \\
& \text { (Mujer 4, } 40 \text { años) }
\end{aligned}
$$

La mayoría de las mujeres informaron que los médicos pedían permiso antes de comenzar los exámenes o procedimientos físicos. Sin 
embargo, algunas señalaron que varias veces habían sido examinadas sin consentimiento expreso por varios profesionales, incluidos estudiantes, de una manera que consideraban excesiva e innecesaria. Aunque los exámenes vaginales durante el parto son rutinarios, pueden ser muy dolorosos y son percibidos por las mujeres indígenas como una violación de su intimidad, por lo que es fundamental que los médicos expliquen detalladamente estos tactos. Si no se obtiene el consentimiento informado, estos exámenes podrían incluso ser vistos como violaciones, tal como lo relata la siguiente participante. "Yo siento que él me abusó sexualmente, porque quedarse cinco minutos haciendo supuestamente tacto... yo siento que me abusaron" (Mujer 1, 43 años). Las mujeres indígenas se sienten particularmente incómodas con estos exámenes, ya que no están acostumbradas a ser tocadas y examinadas por médicos varones, por lo que es necesaria una consulta adecuada y empática con estas mujeres para evitar que se sientan asustadas e incómodas. Además de los exámenes sin consentimiento, las participantes informaron sentirse presionadas a realizar procedimientos u operaciones que no deseaban:

El doctor me dijo: ¿cuántos hijos tiene? y yo "ah dos", "y venga yo le programo la operación", entonces yo le dije: "no, yo no me quiero operar" y me dijo: "ipor qué no se va a operar si ya tiene dos hijos, ¿qué está pensando?", entonces yo le dije: "es que es mi cuerpo y yo decido si me quiero operar o no... es que desde mi cosmovisión no está bien vista la operación", entonces uno me dijo: "ay, si, por eso es que se llenan tanto de hijos y no los pueden ni mantener y hasta piden limosna. (Mujer 6, 32 años)

\section{Atención sin dignidad}

La atención sin dignidad, incluida la manifestación de la violencia obstétrica, descrita por muchos como abuso ${ }^{(36)}$, fue una forma $\mathrm{CO}^{-}$ mún de violencia experimentada por las mujeres de este estudio. Casi todas describieron haber experimentado humillación, estigma frente a sus identidades como mujeres indígenas, expresiones que las culpabilizaban o trato rudo durante el parto. La mayoría de estas experiencias se manifestaron a través de abusos verbales (gritos, regaños o insultos). Varias mujeres señalaron que las enfermeras o los médicos les hablaban de una manera muy brusca durante el parto, con expresiones como "jabra más las piernas!", "para tener relaciones sexuales sí pudo, para quedar embarazada sí pudo", "ay, ya viene el doctor y que no vea todo este sangrero", o incluso "¿usted vino a parir o a cagar?". Otras describieron haber sido "miradas de manera diferente" por sus identidades indígenas, lo que influyó en toda su interacción con los profesionales de salud. Algunas contaron insultos específicos tales como comentarios raciales o expresiones que aludían a las identidades indígenas de las mujeres.

Además de la atención no digna que se manifiesta a través del abuso verbal, muchas mujeres sentían que sus experiencias de parto no eran dignas, debido al propio entorno del hospital. La mayoría de las mujeres de este estudio dieron a luz en hospitales que no permitían visitas (incluido el padre del bebé o, como se mencionó anteriormente, parteras) y en los que todas las mujeres en parto compartían una habitación común y estaban separadas solo por cortinas finas. Así pues, se sentían solas y aisladas durante sus partos, ya que este entorno difería claramente de un parto tradicional en una comunidad indígena con familia, amigos y curanderos tradicionales cerca.

Yo, por ejemplo, no estuve acompañada porque yo quería que mi familia estuviera aquí, que estuviera preparada y yo no estaba preparada para que me encerraran en un hospital... entonces te prohíben, que solo puede entrar una persona, yo quería que mi partera me acompañara y me dijeron: "esto no es posible, eso en este hospital no es posible" y yo: "pero es que es un asunto de llegar bonito, de llegar agradable, de llegar tranquilo. Eso no es un asunto de etnia, esto es un asunto de humanidad". (Mujer 7, 37 años) 


\section{Abuso físico}

Ninguna de las mujeres de este estudio informó haber sido abofeteada o golpeada durante el parto. Sin embargo, varias mujeres experimentaron la maniobra de Kristeller, que rara vez se indica y que ha sido desacreditada repetidamente en la bibliografía médica por ser anticuada y peligrosa ${ }^{(39)}$. Las participantes del estudio experimentaron esta maniobra, que utiliza la presión del fondo uterino durante el parto, como humillante e incómoda:

De montar, eso es lo que no me ha gustado de un parto, montar encima de la mujer que está acostada y la posición de uno estar acostada es algo incómoda ¿sí? Porque esa posición no es de cualquier parto, pues es acostada, no amarrada, acostada y, encima el médico, que lo hagan así y la otra jalando el bebé eso me pareció muy horrible. (Mujer 1, 43 años)

\section{DISCUSIÓN}

En el presente artículo se dan a conocer experiencias de mujeres indígenas que tuvieron sus partos de manera institucionalizada, y donde fue posible identificar diferentes formas de violencia obstétrica. Las mayorías de estas formas de violencia también han sido identificadas en mujeres occidentalizadas ${ }^{(4,40,41)}$; sin embargo, nuevas expresiones emergen cuando la mujer que da a luz tiene una procedencia indígena. Las complejas y múltiples formas en que se presenta este fenómeno llevan a pensar que nos encontramos frente a un asunto que no se explica únicamente a partir de las interacciones entre sujetos, sino que es generado por aspectos sistémicos y estructurales mucho más amplios y complejos, razón por la cual es necesario aumentar sus marcos de comprensión.

Para lograr lo anterior, es menester acercarse a los desarrollos propuestos por algunas ciencias sociales, como la antropología y la sociología. Estos discursos alimentan el campo de la salud, al examinar más de cerca los matices de clase, género, raza y etnia dentro de los actos de violencia y a develar cómo dichas expresiones se inscriben en los cuerpos de las mujeres ${ }^{(42)}$. Las luces que arrojan estas disciplinas permiten sostener que, incluso en un proceso que se espera sea sanador como es el recibir un servicio de salud, se encuentra mediado por el poder, las relaciones asimétricas y la inequidad entre mujeres y hombres.

En el caso particular de la violencia obstétrica, esta ha sido estudiada por diversos investigadores $^{(2,43,44)}$, quienes la describen e interpretan desde diferentes vertientes teóricas. Castro y Erviti, por ejemplo, la comprenden como un habitus autoritario (retomando la categoría de "habitus" de Bourdieu)(45), expresado en posturas represivas que buscan callar los sentires y expresiones de las mujeres frente a su parto, inculparlas ante posibles dificultades que se puedan presentar durante el proceso, o desplegar una posición de pretendida superioridad moral ${ }^{(46)}$. Para estos autores, el orden institucional encarnado en los profesionales de la salud, pone a estos en el lugar protagónico, invistiéndolos de jerarquía y autoridad, en tanto son quienes gozan del saber necesario para que el alumbramiento sea realizado de una manera segura y exitosa. Así las cosas, la mujer queda relegada a un segundo plano, en tanto no sabe, pues no domina el saber médico ${ }^{(46)}$.

Lo anterior también se explica como una forma radical de opresión, que manifiesta la supresión de la expresión y de la valía intelectual y moral de la persona oprimida y que se denomina injusticia epistémica. Este concepto fue aportado por la filósofa británica Miranda Fricker y hace alusión a la forma de discriminación y opresión que consiste en negar validez como conocimiento al saber y experiencia de quienes son considerados entes carentes de capacidad o legitimidad intelectual(47). En estas modalidades de violencia podemos comprender cómo históricamente $y$, en particular, las mujeres indígenas, han sido oprimidas entre los oprimidos y objeto de formas de violencia que destacan por su persistencia. 
Esta descripción del habitus autoritario e injusticia epistémica que exhiben los profesionales de la salud, bien se puede identificar a lo largo de las categorías desarrolladas en los resultados del presente estudio. Por ejemplo, allí donde las mujeres exponen el sentirse ignoradas frente a las dudas, preocupaciones, demandas y saberes sobre la atención que están recibiendo y la experiencia que atraviesa su cuerpo durante el trabajo de parto. Esta forma de ignorar el sentir y saber de la mujer, desprecia su vivencia emocional y comprensiva, relegándola a ser un sujeto pasivo, dedicado a obedecer las órdenes del médico.

Por otra parte, la pretendida superioridad moral que asumen los profesionales de la salud frente a las mujeres que asisten, en el presente estudio se expresa en testimonios de mujeres que describen que, durante su trabajo de parto, recibieron órdenes por medio de las cuales les quisieron imponer decisiones sobre su cuerpo y su vida reproductiva y, peor aún, escucharon expresiones que denotaban claros juicios de valor frente a las decisiones reproductivas que ellas tomaban. Este tipo de expresiones comunican a la mujer que el profesional de la salud tiene autoridad para decidir, opinar y juzgar a la luz de su propia moral, todo aquello que acontezca en el cuerpo y la vida reproductiva de las mujeres que atienden en los servicios de ginecobstetricia.

Ahora bien, sobre el habitus autoritario, se puede decir que son conductas socialmente cultivadas en el personal de salud, y cuyo origen se puede rastrear desde su proceso de formación profesional a través del currículo oculto, en forma de réplica del profesor al alumno, sin atravesar un proceso de reflexión y toma de conciencia. Así las cosas, se instalan como una práctica profesional "naturalizada" en tanto no son percibidas como violentas por los profesionales ${ }^{(46)}$. El hecho de que la mayoría de las participantes del estudio hayan experimentado algún tipo de trato indigno, reflejado en gritos, regaños e insultos, permiten pensar que estas prácticas se encuentran interiorizadas en un número importante de profesionales de la salud y que de ninguna manera son hechos aislados.
La antropóloga Brigitte Jordan arroja otras luces para comprender este fenómeno. Esta autora introdujo el parto como un importante lugar de análisis cultural, al describir el parto como un "acontecimiento universal de crisis vital" que está tan arraigado social y culturalmente como un acontecimiento fisiológico ${ }^{(48)}$. Brigitte Jordan ${ }^{(48)}$ y Margarita Kayhan $^{(49)}$ han dilucidado la ecología del nacimiento como un lugar de gran significado: "El parto, por el mero hecho de estar situado en algún lugar, tiene lugar inevitablemente en el territorio de alguien", afirma Jordan ${ }^{(50)}$ destacando la dinámica de poder implícita que entra en juego cuando las mujeres indígenas dan a luz en los sistemas hospitalarios occidentales. De este modo, se plantean preguntas sobre quién es el "dueño" del parto, quién tiene derecho a tomar decisiones importantes sobre lo que ocurre o qué acontecimientos son normales o patológicos: ¿la mujer o el profesional de salud? Jordan ${ }^{(50)}$ introduce el fenómeno del conocimiento autorizado, señalando que:

...la observación central es que para
cualquier dominio particular existen
varios sistemas de conocimiento, algu-
nos de los cuales, por consenso, llegan a
tener más peso que otros, ya sea porque
explican el estado del mundo mejor para
los propósitos en cuestión (eficacia) o
porque están asociados con una base de
poder más fuerte (superioridad estructu-
ral), y por lo general ambos.

Este fenómeno del conocimiento autorizado es especialmente importante al considerar el parto intercultural. La globalización y la occidentalización han alterado el lugar de la toma de decisiones autorizadas durante el parto, ya que la práctica biomédica ha penetrado en las culturas y ha desplazado a la medicina tradicional, especialmente en las instituciones sanitarias. Esta dinámica cambiante ha provocado choques entre la medicina tradicional y la biomedicina, lo que ha aumentado la división entre estos sistemas y ha hecho que el parto intercultural sea cada vez más difícil de conseguir ${ }^{(51,52,53)}$. 
Ahora bien, la violencia obstétrica no solo puede explicarse por el habitus médico, la injusticia epistémica o el conocimiento autorizado, sino también como resultado del entorno del parto. Para el caso que se analiza, esta se presenta en el marco de un sistema de salud que ha mercantilizado la vida, al fundamentar su lógica en el criterio de rentabilidad. Así lo han denunciado autoras como Laurell(54), quien afirma que, gracias a las orientaciones dadas en la propuesta del Banco Mundial "Invertir en Salud"(55), el enfoque dominante de las políticas de salud adoptado en algunos países de América Latina ha sido el económico, y no la satisfacción de las necesidades de salud de la población. Dicho informe traza líneas para la privatización de los servicios de salud, el traslado de costos a las familias, la desprotección del Estado y, por consiguiente, la mercantilización de la salud(56).

Y todo lo anterior sucede gracias a la legitimación y legalización del mercado por medio de acuerdos y medidas implementadas a partir de la década de 1980 en varios países de la región de las Américas. Tal es el caso de los Planes de Ajuste Estructural (PAE), en los que se argumentó la necesidad de reformar los Estados en el sentido de terminar con el intervencionismo y permitir la presencia del libre mercado. Así las cosas, el derecho a la salud se terminó convirtiendo en una venta de servicios ${ }^{(57)}$.

Esta lógica de mercado ha llevado al cierre de servicios de maternidad bajo el argumento de que son improductivos o poco rentables, llevando a que las salas materno-infantiles, que aún prestan sus servicios, se saturen y colapsen, haciendo inoperante su atención y sobrecargando laboralmente a los profesionales de salud ${ }^{(56)}$. Bajo esta perspectiva, se ha trivializado la vida, lo que posibilita que se presenten situaciones como las narradas por algunas mujeres participantes del estudio, a quienes se les negó el ingreso a los servicios de salud bajo el argumento de que no se encontraban afiliadas a una Entidad Promotora de Salud, o no había camas disponibles.

Ahora bien, el habitus médico autoritario, la injusticia epistémica, el conocimiento autorizado y la mercantilización de la vida ayudan a explicar algunas formas de violencia obstétrica padecida por las mujeres en las salas de parto; pero otras formas de violencia, como el estigma y la falta de respeto frente a los saberes y prácticas ancestrales o representaciones de mundo diferentes al occidental, requieren otro tipo de análisis, como el que propone Quijano sobre la colonialidad $^{(58)}$. Este autor explica la colonialidad como una forma de imponer una clasificación de la población mundial a partir de un criterio racial/étnico, el cual opera en cada ámbito, material y subjetivo de la existencia cotidiana y a escala social ${ }^{(58)}$. Es una imposición sobre el dominio del conocimiento, el trabajo, la autoridad y las relaciones intersubjetivas, mediados por la idea de raza(59), y que ha configurado una estructura de pensamiento Ilamada "moderna y racional", en la que las comprensiones del mundo que se diferencien son asumidas como anticuadas y ajenas a la razón ${ }^{(59)}$.

Adicionalmente, esta forma de asumir la mujer indígena con la impronta de la colonialidad genera una serie de microagresiones. Este término comenzó como una forma de describir las experiencias comunes de abusos recibidos por los afroamericanos ${ }^{(60)}$ y fueron categorizadas en tres tipos: micro aprensiones (acciones despectivas verbales o no verbales), micro insultos (interacciones groseras o insensibles) y micro invalidaciones (interacciones que niegan, descartan o anulan el conocimiento). Aunque estas acciones pueden parecer relativamente menores, como forma de abuso, expresan una forma encubierta de racismo que a menudo es ambigua, nebulosa y, en consecuencia, más difícil de identificar y denunciar ${ }^{(61)}$.

Así, el fenómeno de la colonialidad, que permea la manera en que hablamos, pensamos y sentimos ${ }^{(58)}$, podría explicar el hecho de que, frente a mujeres que aún conservan en su representación del mundo otras formas no occidentalizadas de concebir el parto, los profesionales de salud no encuentren en sus discursos saberes que aporten al proceso de alumbramiento, ni identifiquen en sus prácticas reproductivas acciones Ilenas de significados y sentidos para la vida de 
dichas mujeres y sus comunidades. Como las mujeres indígenas tienen otros saberes y prácticas, que no caben en la lógica de la formación médica occidental, cuando estas emergen durante la atención al parto, son silenciadas y estigmatizadas a manera de microagresiones, como recurso que le queda al profesional de la salud frente a un saber que le es extraño y que viene de un sujeto no modernizado, ni occidentalizado y que se identifica con una etnia que desde la colonia ha sido subalternizada.

Los resultados de la presente investigación permitieron identificar formas de violencia obstétrica ya descritas en otras investigaciones cuya población de interés fueron mujeres occidentalizadas. Dichas formas de violencia pueden ser explicadas por un habitus médico autoritario y el conocimiento autorizado que históricamente ha hecho carrera en el campo de formación de las diversas disciplinas de la salud, permeando las prácticas, pero no necesariamente la conciencia y reflexión de los profesionales, lo que en última instancia termina reproduciendo injusticias epistémicas. Adicionalmente, esta forma de violencia se desarrolla en un sistema de salud que ha privilegiado las lógicas del mercado por encima de los principios que protegen la salud y la vida. Estos elementos configuran unas condiciones que posibilitan la emergencia de una atención que desconoce los intereses, necesidades y dignidad de las mujeres.

Sin embargo, el origen de la mujer indígena la pone en una condición más susceptible aún, pues además de experimentar todas las formas de violencia obstétrica reportadas por las mujeres occidentalizadas, padecen otras que se explican desde su etnia: el estigma e irrespeto de sus prácticas y saberes ancestrales. Lo anterior se puede comprender desde el proceso de colonialidad vivido por los pueblos originarios, y que hoy en día se expresa, en el contexto de la atención al parto, bajo formas de microagresiones como el desconocimiento y desprecio de los saberes ancestrales que las mujeres de las comunidades indígenas aún conservan.

\section{FINANCIAMIENTO}

Este artículo es un producto parcial del proyecto " $L a$ salud materna y la violencia obstétrica: las experiencias de las mujeres emberas en Medellín - Colombia, 20192020", financiado por la beca de la Comisión Fulbright de Colombia 2019-2020; y por el Grupo de Investigación en Salud Mental (GISAME), a través de la Estrategia para la Sostenibilidad 2020-021 del Comité para el Desarrollo de la Investigación (CODI) de la Universidad de Antioquia, código ES84190026.

\section{AGRADECIMIENTOS}

Agradecemos a todas las mujeres participantes del estudio, a la Organización Indígena de Antioquia (OIA) y a Jobany Tascón.

\section{CONFLICTO DE INTERESES}

Las autoras declaran no tener vínculos o compromisos que condicionen lo expresado en el texto y que puedan ser entendidos como conflicto de intereses.

\section{REFERENCIAS BIBLIOGRÁFICAS}

1. Organización Mundial de la Salud. Sexual health, human rights and the law [Internet]. Ginebra: OMS 2015 [citado 28 jun 2020]. Disponible en: https://tinyurl. com/yrzf9adz.

2. Belli LF. La violencia obstétrica: otra forma de violación a los derechos humanos. Revista Red Bioética. 2013;1(7):25-34. 
3. Fondo de Población de las Naciones Unidas. Ley Orgánica sobre el derecho de las mujeres a una vida sin violencia [Internet]. 2007 [citado 4 feb 2021]. Disponible en: https://tinyurl.com/543chzu8.

4. Mejía Merino CM, Zapata Alvarez LF, Molina Berrío DP, Arango Urrea JD. Dehumanization during Delivery: Meanings and Experiences of Women Cared for in the Medellín Public Network. Investigación y Educación en Enfermería. 2018;36(1):e03. doi: http://dx.doi. org/10.17533/udea.iee.v36n1e03.

5. Otis KE, Brett JA. Barriers to hospital births: why do so many Bolivian women give birth at home? Revista Panamericana de Salud Pública. 2008;24(1):46-53. doi: 10.1590/s1020-49892008000700006.

6. Population Reference Bureau. Meeting the Reproductive Health Needs of Displaced People [Internet]. 2002 [citado 15 may 2019]. Disponible en: https:// tinyurl.com/a56u2487.

7. Argüello-Avendaño H, Mateo-González A. Parteras tradicionales y parto medicalizado, ¿un conflicto del pasado? Evolución del discurso de los organismos internacionales en los últimos veinte años. LiminaR. 2014;12(2):13-29.

8. Moreno Sierra PE, Guzmán Castillo KA. Haciendo visible lo invisible: prácticas comunes innecesarias como expresión de violencia obstétrica [Tesis de Maestría]. Bogotá: Pontificia Universidad Javeriana; 2017.

9. Sadler M, Santos MJ, Ruiz-Berdún D, Rojas GL, Skoko E, Gillen P, et al. Moving beyond disrespect and abuse: addressing the structural dimensions of obstetric violence. Reproductive Health Matters. 2016;24(47):47-55. doi: 10.1016/j.rhm.2016.04.002.

10. Bohren MA, Vogel JP, Hunter EC, Lutsiv O, Makh SK, Souza JP, et al. The mistreatment of women during childbirth in health facilities globally: A mixed-methods systematic review. PLoS Medicine. 2015;12(6):e1001847. doi: https://doi.org/10.1371/journal.pmed.1001847.

11. Valdez-Santiago R, Salazar-Altamirano Y, RojasCarmona A, Arenas-Monreal L. El abuso contra las mujeres durante la atención del parto en hospitales públicos en México. Revista Conamed. 2016;21(1):42-47.

12. Restrepo-Sánchez A, Rodríguez-Martínez D, TorresCastro NM. Me des-cuidaron el parto: la violencia obstétrica y el cuidado recibido por el personal de la salud a mujeres durante su proceso de parto. [Tesis de grado]. Bogotá: Pontificia Universidad Javeriana; 2016.

13. CHIRAPAQ. Informe sobre maltrato y violencia contra las mujeres indígenas durante la atención de salud reproductiva, con enfoque en parto [Internet]. 2019 [citado 5 sep 2021]. Disponible en: https://tinyurl. com/3uubfbje.

14. Rangel-Flores YY, Martínez-Ledezma A. Ausencia de percepción de violencia obstétrica en mujeres indígenas del centro norte de México. Revista Conamed. 2017;22(4):166-169.
15. Sosa Sánchez IA. Estratificación de la reproducción y violencia obstétrica en servicios públicos de salud reproductiva. Alteridades. 2018;28(55):87-98.

16. Muelas Izquierdo DK. Partería: perspectivas jurídicas de los conocimientos tradicionales y los derechos sexuales y reproductivos de las mujeres Iku. Bogotá: Universidad Colegio Mayor de Nuestra Señora del Rosario; 2018.

17. Foro Internacional de Mujeres Indígenas. Diálogo de saberes sobre la violencia contra las mujeres indígenas aproximaciones metodológicas a la investigación intercultural [Internet]. 2013 [citado 17 oct 2020]. Disponible en: https://tinyurl.com/nrvjs5ap.

18. El Tiempo. Medellín es hogar de cerca de 3.000 indígenas [Internet]. 2017 [citado 14 feb 2020]. Disponible en: https://tinyurl.com/k8nz9fju.

19. Ministerio de Cultura de Colombia. Emberá [Internet]. s/f [citado 17 oct 2020]. Disponible en: https:// tinyurl.com/3y6rn5s6.

20. Pernía Domicó K, Angel Domicó L, Jaramillo E. Movilización cultural del pueblo Emberá Katío del Alto Sinú [Internet]. 2001 [citado 24 feb 2020]. Disponible en: https://tinyurl.com/y7zktjrh.

21. Bula Romero JA, Maza Padilla LE, Orozco Valeta M. Prácticas de cuidado cultural en el continuo reproductivo de la mujer Emberá Katio del Alto Sinú. Enfermería: Cuidados Humanizados. 2019;8(1):102-138. doi: http://dx.doi.org/10.22235/ech.v8i1.1785.

22. Pueblos Originarios. El universo Emberá y el Jaibanismo. [Internet]. 1988 [citado 24 feb 2020]. Disponible en: https://tinyurl.com/c495w7f8.

23. Arias-Valencia MM. Comportamiento reproductivo en las etnias de Antioquia-Colombia. Salud Pública de México. 2001;43(4):268-278.

24. Draper J. Ethnography: Principles, practice, and potential. Nursing Standard. 2015;29(36):35-41. doi: 10.7748/ns.29.36.36.e8937.

25. Glaser BG, Strauss AL. The discovery of grounded theory: Strategies for qualitative research. Chicago: Aldine Publishing; 1967.

26. Sabino C. El proceso de investigación. Bogotá: Editorial Panamericana; 1992.

27. Organización Indígena de Antioquia. Contexto histórico OIA. [Internet]. 2021 [citado 9 mar 2021]. Disponible en: https://tinyurl.com/bvjdhs3h.

28. Hernández Sampieri R, Fernández Collado C, Baptista Lucio P. Metodología de la Investigación. 5ta ed. México DF: McGraw-Gill; 2010.

29. Saunders B, Sim J, Kingstone T, Baker S, Waterfield J, Bartlam B, Burroughs H, Jinks C. Saturation in qualitative research: exploring its conceptualization and operationalization. Quality \& Quantity. 2018;52(4):1893-1907. 
30. Bernard HR, Wutich A, Ryan GW. Analyzing qualitative data: Systematic approaches. Thousand Oaks: SAGE; 2016.

31. Roulston K, Shelton SA. Reconceptualizing bias in teaching qualitative research methods. Qualitative Inquiry. $2015 ; 21(4): 332-342$.

32. Strauss A, Corbin J. Bases de la Investigación cualitativa: Técnicas y procedimientos para desarrollar la teoría fundamentada. Medellín: Editorial Universidad de Antioquia; 2002.

33. Bourgeault I, Dingwall R, De Vries R. The SAGE handbook of qualitative methods in health research. Los Angeles: SAGE; 2010.

34. Asociación Médica Mundial. Declaración de Helsinki de la AMM: Principios éticos para las investigaciones médicas en seres humanos [Internet]. 2017 [citado 9 mar 2021]. Disponible en: https://tinyurl. com/2zyspaad.

35. Colombia, Ministerio de Salud. Resolución 8430 de 1993 por el cual se establecen las normas científicas, técnicas y administrativas para la investigación en salud [Internet]. 1993 [citado 24 feb 2020]. Disponible en: https://tinyurl.com/f699rb5u.

36. d'Oliveira AFPL, Diniz SG, Schraiber LB. Violence against women in health-care institutions: an emerging problem. The Lancet. 2002;359(9318):1681-1685. doi: https://doi.org/10.1016/S0140-6736(02)08592-6.

37. Bowser D, Hill K. Exploring evidence for disrespect and abuse in facility-based childbirth: Report of a Landscape Analysis [Internet]. 2010 [citado 9 mar 2021]. Disponible en: https://tinyurl.com/3mhzmny6.

38. Castro R, Erviti J. Violations of reproductive rights during hospital births in Mexico. Health Hum Rights. 2003;7(1):90-110. doi: https://doi.org/10.2307/4065418.

39. Rengel Díaz C. La maniobra de Kristeller: revisión de las evidencias científicas. Matronas Profesión. 2011; 12(3):82-89.

40. Briceño Morales X, Enciso Chaves L, Yepes Delgado C. Neither medicine nor health care staff members are violent by nature: Obstetric violence from an interactionist perspective. Qualitative Health Research. 2018;28(8):1308-1319. doi: $10.1177 / 1049732318763351$

41. Canevari Bledel C. Cuerpos enajenados: experiencias de mujeres en una maternidad pública. Santiago del Estero: Facultad de Humanidades, Ciencias Sociales y Salud, UNSE; 2011

42. Zacher Dixon L. Obstetrics in a time of violence: Mexican midwives critique routine hospital practices. Medical Anthropology Quarterly. 2015;29(4):437-454. doi: 10.1111/maq.12174.

43. Aguiar J, Lucas-d'Oliveira A, Blima-Shraiber L. Violência institucional, autoridade médica e poder nas maternidades sob a ótica dos profissionais de saúde. Ca- dernos de Saúde Pública. 2013;29(11):2287-2296. doi: https://doi.org/10.1590/0102-311×00074912.

44. Pozzio M. La gineco-obstetricia en México: entre el "parto humanizado" y la violencia obstrétrica. Estudios Feministas. 2016;24(1):101-117.

45. Bourdieu P. El sentido práctico. Buenos Aires: Siglo XXI Editores; 2007.

46. Castro R, Erviti J. El habitus en acción: la atención autoritaria del parto en los hospitales. En: Sociología de la práctica médica autoritaria: violencia obstétrica, anticoncepción inducida y derechos reproductivos. Cuernavaca: Centro Regional de Investigaciones Multidisciplinarias, Universidad Nacional Autónoma de México; 2015. p. 81-135.

47. Fricker M. Injusticia epistémica. Barcelona: Herder Editorial; 2017.

48. Jordan B. Birth in four cultures: A crosscultural investigation of childbirth in Yucatan, Holland, Sweden and the United States. California: Eden P Women's Publications; 1978 .

49. Kay MA. Anthropology of human birth. Bloomington: F.A. Davis; 1982.

50. Jordan B. Authoritative knowledge and its construction. In: Davis-Floyd RE, Sargent CF, eds. Childbirth and authoritative knowledge: Cross-cultural perspectives. Berkeley: University of California Press; 1997. p. 55-79.

51. Sargent C, Bascope G. Ways of knowing about birth in three cultures. Medical Anthropology Quarterly. 1996;10(2):213-236. doi: 10.1525/maq.1996.10.2.02a 00070 .

52. Davis-Floyd RE, Sargent CF, eds. Childbirth and authoritative knowledge: Cross-cultural perspectives. Berkeley: University of California Press; 1997.

53. Strong AE. The Maternity ward as mirror: Maternal death, biobureaucracy, and institutional care in the Tanzanian health sector. [Tesis de doctorado]. Saint Louis: Graduate School of Arts and Sciences, Washington University; 2017.

54. Laurell A. La segunda reforma de salud: Aseguramiento y compra venta de servicios. Salud Colectiva. 2010;6(2):136 148. doi: https://doi.org/10.18294/sc.2010.362.

55. Banco Mundial. Invertir en Salud: Informe sobre el desarrollo mundial. Washington DC: Banco Mundial; 1993.

56. Arango-Urrea J, Molina-Berrío D, Mejía-Merino C, Zapata L. La atención a las madres durante el proceso de parto en algunos servicios de salud de la ciudad de Medellín: un acontecimiento enmarcado en el neoliberalismo y la mercantilización de la vida. Revista Gerencia y Políticas de Salud. 2018;17(35):1-17. doi: https://doi. org/10.11144/javeriana.rgps17-35.amdp.

57. Moya Cisneros CA. La mercantilización y lista de espera de la salud en Costa Rica. Cátedra. 2020;(17):156169. 
58. Quijano A. Colonialidad del poder y clasificación social. En: Cuestiones y horizontes: de la dependencia histórico-estructural a la colonialidad/descolonialidad del poder. Buenos Aires: CLACSO; 2014.

59. Ortiz-Ocaña A, Arias M, Pedrozo ¿Qué es la Colonialidad? En: Decolonialidad de la educación: Emergencia/urgencia de una pedagogía decolonial. Santa Marta: Editorial Unimagdalena; 2018. p. 18-36.
60. Williams MT. Microaggressions: clarification, evidence, and impact. Perspectives on Psychological Science. 2020;15(1):3-26.

61. Smith-Oka V. Microaggressions and the reproduction of social inequalities in medical encounters in Mexico. Social Science \& Medicine. 2015;143:9-16. doi: 10.1016/j.socscimed.2015.08.039.

\section{FORMA DE CITAR}

Gleason EG, Molina Berrío DP, López Ríos JM, Mejía Merino CM. "Parir no es un asunto de etnia, es un asunto de humanidad": experiencias frente a la violencia obstétrica durante la atención al parto en mujeres indígenas. Salud Colectiva. 2021;17:e3727. doi: 10.18294/sc.2021.3727.

Recibido: 27 jul 2021 | Versión final: 23 sep 2021 | Aprobado: 4 nov 2021 | Publicado en línea: 23 nov 2021



Esta obra está bajo una licencia Creative Commons Atribución 4.0 Internacional (CC BY 4.0). Atribución - Se debe dar crédito de manera adecuada, brindar un enlace a la licencia, e indicar si se han realizado cambios. Puede hacerlo en cualquier forma razonable, pero no de forma tal que sugiera que usted o su uso tienen el apoyo de la licenciante. Sin restricciones adicionales — No se pueden aplicar términos legales ni medidas tecnológicas que restrinjan legalmente a otras personas a hacer cualquier uso permitido por la licencia. 